\title{
Ubiquitous Healthcare: Do we want it?
}

\author{
Linda Little \\ PaCTLab \\ Northumbria University \\ Newcastle upon Tyne \\ \#441912437250 \\ I.little@northumbria.ac.uk
}

\author{
Pam Briggs \\ PaCTLab \\ Northumbria University \\ Newcastle upon Tyne \\ \#44 1912274570 \\ p.briggs@northumbria.ac.uk
}

\begin{abstract}
In this paper we describe the development and test of a futuristic health scenario that allows the seamless exchange of sensitive personal data. The scenario was used to elicit user attitudes and concerns in thirty-eight focus groups drawn from a representative population sample. Emergent themes are described in terms of firstly, those hygiene factors that act as precursors to successful engagement with the technology, secondly, those motivators that would drive acceptance and thirdly, longer-term societal impact.
\end{abstract}

\section{Categories and Subject Descriptors}

K 4 Computers and society, K 4.1 Computer related health issues, privacy

\section{General Terms}

Management, Design, Reliability, Security, Human Factors,

\section{Keywords}

Ubiquitous computing, information exchange, health, scenarios

\section{INTRODUCTION}

Inevitably new technologies change social practices and norms and so one of the goals of HCI research is to assess the social impact of computer-based technologies [3]. One area in which social impact predictions are difficult is in the field of ubiquitous computing, where it is difficult for potential users to imagine the extent to which 'invisible' computational devices may capture and ultimately influence behaviour. We describe a study in which we engaged with key stakeholders in order to develop a futuristic ubicomp scenario describing the management and exchange of health information This scenario was subsequently used to elicit social attitudes and trust and privacy concerns, following Streitz \& Nixon's [4] argument that security, privacy, and trust are the critical issues for ubiquitous systems development.

The health context is particularly interesting as health records contain highly personal content, much of which is extremely sensitive (e.g. treatment for a sexually transmitted disease). Established technologies are already generating privacy concerns around the issue of electronic patient records, where, for example, numerous cases of abuse have been reported [2] and so it is hardly surprising that privacy has been

(C) The Author 2008 .

Published by the British Computer Society placed firmly on the agenda in any discussion of ubiquitous health monitoring.

\section{METHOD}

We developed a ubicomp health scenario that was scripted and filmed in keeping with a methodology found to be very effective in the elicitation of social attitudes and rules [1]. Firstly, key stakeholders drawn from industry and academia were asked to capture the key elements in a futuristic health interaction. This information was then given to a professional playwright who created a short script, capturing all of the salient information. The script was handed over to a media production company for filming and the production was overseen by both the producer and the research team to ensure correct interpretation. British Sign Language (BSL) and subtitles were also added to a master copy of the film for use in groups where participants had various visual or auditory impairments. The scenario is described below.

Bob is in his office talking on his personal digital assistant (PDA) to a council planning officer with regard to an important application deadline. Built into his PDA are several personalised agents that pass information seamlessly to respective recipients. A calendar agent records and alerts Bob of deadlines, meetings, lunch appointments and important dates. As Bob is epileptic his health agent monitors his health and can alert people if he needs help. An emergency management agent takes control in situations when a host of different information is needed; this agent has the most permissions and can contact anyone in Bob's contact list.

Bob is going to meet his friend Jim for lunch when he trips over a loose paving slab. He falls to the ground and loses consciousness. His health agent senses something is wrong and beeps, if Bob does not respond by pressing the appropriate key on the PDA the agent immediately informs the emergency services. Within seconds the emergency services are informed of Bob's current situation and his medical history. An ambulance is on its way. Paramedics arrive, examine Bob and then inform the hospital of Bob's condition on their emergency device. The hospital staff are now aware of Bob's medical history and his present state, therefore on arrival he is taken straight to the $x$-ray department. $A$ doctor receives the x-rays on her PDA. After examining Bob she confirms that he has a broken ankle, slight concussion and needs to stay in hospital overnight. After receiving treatment Bob is taken to a ward. His emergency management agent contacts John (Bob's boss) of his circumstance. The emergency management agent transfers the planning application files to John's PDA so the company do not miss the deadline. The agent also informs his parents letting them know his current state of health, exactly where he is so they can visit and that his dog needs to be taken care of. As Bob is also head coach at a local running club the agent informs the secretary Bob will not be attending training the following week. The secretary only receives minimal information through the permissions Bob has set.

The filmed scenarios were shown to 38 focus groups involving a total number of 304 participants drawn from all sectors of society in the Newcastle upon Tyne area of the UK, including representative groups from the elderly, the disabled and from different ethnic sectors. 
Demographic characteristics of all participants were recorded related to: age, gender, disability (if any), level of educational achievement, ethnicity, and technical stance. Although several participants with physical disabilities attended the main group sessions two group sessions for people with visual and auditory impairments were carried out at the Disability Forum in Newcastle. The forum was considered to have easier access and dedicated facilities for people with such disabilities.

On recruitment all participants received an information sheet that explained the study and the concept of ubicomp technologies. Participants were invited to attend Northumbria University, UK to take part in a group session. The groups were run at various times and days over a three-month period. At the beginning of each group session the moderator gave an explanation and description of ubicomp technologies. After the initial introduction the filmed health scenario was shown (accompanied by three other scenarios depicting different contexts, but not reported here) and attitudes elicited. The duration of the sessions was approximately ninety minutes.

\section{RESULTS AND DISCUSSION}

All group discussions were transcribed and a sentence-bysentence analysis was employed using the Atlas.ti ${ }^{\mathrm{TM}}$ qualitative software program. Two members of the research team coded and compared the data for consistency, good inter-rater reliability was found. Several key constructs related to disclosure, privacy, trust, usability and social issues associated with the use of a ubicomp system emerged. These constructs were found to be interrelated and multidimensional. A representation of these constructs, reflecting Hertzberg et al's (1959) theory of motivation (in terms of hygiene factors and motivators) is given below in Table 1 alongside a number of additional constructs in relation to societal impact. The emergent themes are then each described in some detail below.

\begin{tabular}{lll}
\hline Hygiene Factors & Motivators & Societal Impact \\
\hline Credible & Healthcare & Over-reliance \\
Secure & Convenience & Dehumanisation \\
Reliable & & Bystander apathy \\
Accurate & & Social isolation \\
Transparent & & Enforced participation \\
Context aware & & \\
Personalised & & Profile abuse \\
Easy to use & & Surveillance \\
& & Health risks \\
& & Environmental \\
& & concerns \\
\hline
\end{tabular}

\section{Costs}

Table 1: Key constructs associated with use of an ubicomp system for Health.

\section{Hygiene Factors}

We define hygiene factors as those factors that must be in place in order for users to feel comfortable in the adoption of a new system, but they are not in and of themselves motivators to use the system. Effectively, in order to be acceptable, a health ubicomp system must be:

a) Credible
Participants who visited their GP and/or hospital consultant on a regular basis discussed access and exchange of health information in terms of the credibility of the physician. They raised concerns over the extent to which a ubicomp system could ever be as credible.

'It's not trusting organisations to use that information for my best interest.'

'If you could do it through something like the BBC because it's typically British, you are going to trust the $B B C$, it's always been there, it's something tangible, but for a lot of older people, it's new and it's different, you know they don't trust it, whereas they trust their television because they have watched it all of their life.'

\section{b) Secure}

How secure would ubicomp systems be in exchanging and storing health information? Fraudulent use, hacking, access by third parties, leakage and storage of information were all areas discussed. Participants agreed that being able to verify and access information stored on systems was needed.

'They're not as security conscious as I would like them to be. I don't like the security part of it It's time saving yes and helpful like and you not having to think for yourself.'

\section{c) Reliable}

Pariticpants discussed the relaibility of the system. For example, if the machine malfunctioned and the user was unaware of this what would the consequences be?

'The greater worry I think is that because you have then got a health system taking care of Bob on the basis of the information held in the system, is how correct is that, is the veracity of that information, because if there was a mistake in that information, then things could go awfully wrong...you would worry that there were going to be pieces of information that might be false, that people are acting upon.'

\section{d) Accurate}

Discussion highlighted concerns about human fallibility in keeping systems updated, entering the correct data and setting access preferences.

'So it is all about the information, is all this information accurate or will they make mistakes? You know will it be useful? Some of it maybe is good and some of it not. So I don't know for other people or for myself if RFID would be accurate information. I don't think the information will be a hundred percent accurate.'

\section{e) Transparent}

Participants commented systems needed to be transparent and accessible so information could be verified and changed. Participants acknowledged stakeholders already hold information about you that you are unaware of and this should be made more transparent.

'I mean they don't really know where the information is going and what individuals are actually accessing it or is it just completely churned up by computers? I don't even know but the information is going somewhere and the customer, the consumer should actually have, be allowed to know where that information is going and it should be an open process, open to the consumer, if the consumer wants to know of course, some people might not want to know, but if the consumer wants to know how all that information is processed it should be open.'

\section{f) Context aware}

Human behaviour is unpredictable so could ubicomp systems be truly context aware? Participants agreed that changing preferences to take into account different health contexts might be challenging.

'Because if it makes a decision for you and you think to yourself, I've changed my mind, I'm not in the mood for that and therefore you have mucked your system up on your computer thing and you have to go in 
and tell it I've changed my mind, I don't want to do this, I want to change that.'

'It's just I don't want technology to structure my life, I don't want it telling me know what to do, I don't want it telling other people what I will probably do next ... I want to be spontaneous and intelligent and articulate and respected and react with people not machines.'

Discussion revealed participants concerns over systems being truly sensitive to circumstances under which health information could legitimately be exchanged. Would the system only reveal what information was appropriate at that moment in time?

'I mean there are various aspects of medical information which are protected and it would not be necessary for everybody or for even for emergency services to be aware or anybody in emergency services to be aware of say for example if someone was HIV Positive and okay on treatment it's not necessary for the x-ray department for example to know that, if he is going along for an x-ray of his ankle, but that sort of thing so the next question is yes is there any data protection information that's going, that's floating round in that way so yes, I must say I would be a little bit anxious if information is flowing in a free and uncontrolled way, if it was a really good system, I think safeguards could possibly be built in.'

\section{g) Personalised}

Personalisation was discussed in terms of allergy alerts and reminding people to take medication. Discussion focused on the confidential nature of health information and that the system and stakeholder required sensitivity regarding sending and receiving health data.

'I do think the hospital should have access to your information so say, If I do have a week heart, that should be able to convey to the hospital that plus your entire medical record.'

\section{h) Easy to use}

Participants discussed concern over the complexity of ubicomp systems noting that the programming of preference information might be time-consuming. Discussion also focused on exclusion issues, particularly in relation to age differences in technology use, experience and familiarity.

'New technology for older people is always difficult to comprehend.'

'To the younger generation they have been brought up with that technology. What about the minority groups, disabled, etc?'

'The thought of my Dad using that would cause more cognitive problems rather than solve them. It all depends on your technical ability to start off with.'

\section{Motivators}

Motivators relate to those things that are perceived to bring tangible benefits and that people would strive to achieve.

\section{a) Better healthcare}

Participants recognised that ubicomp systems for exchanging health information could be advantageous, particularly for people with serious medical conditions. Advantages included the possibility of allergy alerts and health professionals having immediate access to patient records when needed. Stakeholder benefits were discussed in terms of monitoring, immediate access and updating patient records and marketing.

'I'm just thinking about the benefits of it you know like, you know the way things work now, I mean the only benefit I would say now is electronic exchange of information that the doctor or hospital sees.'

\section{b) Convenience}

All participants agreed the mobility of ubicomp systems was advantageous and that through diffusion, adoption would probably occur. Participants discussed ubicomp systems in terms of convenience related to their own use and the stakeholder.
I mean I do think that having all the information in one place and an exchange of information and the doctor and the hospital and maybe even the ambulance service being able to forward the information is $\operatorname{good}$....'

\section{Social Impact}

A number of social concerns were raised about the widespread adoption of health ubicomp systems and these are captured below.

\section{a) Over-reliance}

Participants discussed relying too much on the system and/or themselves to exchange information and the responsibility associated with this as very problematic.

'I mean whose life is that busy they have to have a machine to do everything for them?'

'The other thing is if you actually hand over all responsibility to automated systems you know if they make a mistake in your calculation and you are not actually paying any attention, you are just trusting this, you know it is essentially dis-empowering you.'

\section{b) Dehumanisation}

Participants found the concept of ubicomp and the use of agent systems as dehumanising (in the scenario used in this study agent systems were portrayed with human-like features). Participants commented they would not trust such systems and found the concept very impersonal.

'They are pretending to be human. Why not be up front, this is the computer speaking, not this rather twee, dark haired person, who is able to blink.'

'It's all this de-humanisation is how I see it. Do his parents really want to know that he has had an accident, by? Why can they not wait until he can tell them himself? ... Do we have to have everything working like clockwork?'

\section{c) Bystander apathy}

Participants expressed concern in relation to the ways in which the new technologies might change people's lives. Reference was made to ubicomp systems making people lazy, lowering cognitive capacity, reducing work ethic and increasing apathy.

.. if you expected that everybody was like that and someone collapsed in the street, would it stop you going to help them, because you thought oh well the paramedics will be here in a minute, I'm not going to bother!'

'Are we going to exist without this type of technology? I mean are you going to be able to have a day to day life without this technology?'

'Yeah, well it's all about, I think you've picked it up it's all about talking about it before, it's all going too far, this with people's business. I mean I'm not a totally private person, don't get me wrong, I'm quite open a lot of things but some things you just want in your own head and you don't want in the head of a microchip of a machine or whatever you want to call it.'

\section{d) Social isolation}

Discussion highlighted how use of ubicomp systems would result in less human-human interaction and this was considered very problematic.

'Yes you are losing contact with people if you are going to be somebody sat in a room by themselves with a machine like that, talking to people on this internet kind of thing, but there's no substitute for human contact. Its wonderful discourse with human beings face to face rather than through a machine I think.'

'We are so anti-social anyway ... I mean I communicate with a lot of my friends now by text messages whereas before you would have called to them or you know send an email but I see less of people that I care about because it's more convenient to send them a text or an email and 
I hate it, I really do hate it and I think that's going to encourage more because then you're not even going to have to make the effort to send the text message, your machine is going to be sending them a text message because you're overdue writing to them, it's just.'

\section{e) Enforced participation}

Social pressure to conform in using ubicomp was seen as an issue, with many participants believing that little or even no choice would exist. There were fears that forced choice would become the norm. Participants expressed concern that those people who didn't wish to take part would face exclusion from key services.

'I think people who join are going to be pressurised into it. You know when there are facilities there and it gets a little bit pushed and all their friends are doing it and all of their family is doing it.'

'Are we being subtly manoeuvred into doing things that actually, given our own freedom without technology, we wouldn't do, so therefore are we being manipulated in some way into being people that we actually want to become without it?'

\section{f) Profile abuse}

Concerns were raised over the probability of a proliferation of organisations with access to detailed personal information. The concept of profile abuse was a major concern for all participants.

'It could be that you have got a specific condition. You could have a drink problem or whatever and that could get back to your employer or it could get back, you know what I mean, I would have serious concerns, not that I've got a drink problem, not yet anyway!'

\section{g) Surveillance}

Surveillance was a major social issue - potentially beneficial for some people with certain medical conditions, but generally distasteful and associated with a 'big brother' mentality.

'It could work against you like at work for checking what you are doing and everything. Will your boss know what you are doing outside of work?'

'You see all that information where is it going? And even if you say no I don't want you to pass my details on you never really know do you?'

'Yes, the technology side is perfect, it is who controls it.'

\section{h) Health risks and environmental issues}

Participants discussed concerns over health risks and environmental issues related to living in an ubicomp society. Participants referred to problems with radiation from the systems and the global impact of such use.

'Also we are in a time when we are starting to think more and more about the materials we use and the amount of energy we are using and whether we shouldn't be thinking as humans how we should use our energy to think better, write lists rather than use the technology there.'

\section{CONCLUSION}

We have identified a number of hygiene factors related to trust, privacy and secure access information that must be in place before users would feel comfortable with ubicomp systems for health. We have also recognized the importance of two key motivators: improved healthcare and greater convenience in driving people towards acceptance of such new systems. However, perhaps most tellingly, our participants have expressed a number of larger scale societal concerns, not least around the idea that individuals have relatively little long-term choice in the adoption of new technologies. Such societal concerns are worthy of further investigation.

\section{REFERENCES}

[1] Little, L., Briggs, P., \& Coventry, L. (2004). Videotaped Activity Scenarios and the Elicitation of Social Rules for Public Interactions. BHCIG Conference, Leeds, September 2004

[2] Rohm, A.J. \& Milne, G.R. (2004). Just what the doctor ordered: The role of information sensitivity and trust in reducing medical information privacy concern. Journal of Business Research 57 (9), 1000-1011

[3] Shneiderman, B., \& Rose, B. (1997) Social impact statements: Engaging public participation in information technology design in B. Friedman, B. (Ed), Human Values and the Design of Computer Technology, Cambridge Univ. Press (1997), 117-133.

[4] Streitz, N., \& Nixon, P. (2005). The disappearing computer. Communication of the ACM, 48, 3, 32-35 